\title{
HOBBES ET LE RÉPUBLICANISME
}

\author{
Jean TERREL
}

RésumÉ : Hobbes critique la théorie du gouvernement mixte sans le présenter comme républicain. La critique du républicanisme est chez lui la critique de la définition de la liberté comme participation des citoyens à la délibération sur les affaires communes. Elle est pour l'essentiel argumentée dans les Éléments de la loi naturelle et politique et le De cive. Le Leviathan résume et durcit cette critique, tout en développant de façon nouvelle l'idée d'une identité d'essence de la souveraineté dans toutes les sociétés civiles. Cette idée contredit la thèse républicaine selon laquelle il y aurait une différence de nature entre les régimes libres et ceux qui ne le sont pas et permet de constituer en modèle la république romaine ou la démocratie athénienne.

MoTs-CLÉs : Hobbes, républicanisme, gouvernement mixte, souveraineté, citoyen.

ABSTRACT : Hobbes criticizes the theory of mixed government without presenting this mixed government as republican. For him criticizing republicanism means criticizing the definition of freedom as the participation of citizens in the debate on matters concerning them all. We can find the main points of this criticism in the Elements of law natural and politic and in the De cive. In Leviathan Hobbes summarizes this criticism and makes it more dogmatic, while developing in a new way the idea that in all regimes there is an identity of essence in sovereignty. This idea is at variance with the republican thesis stating a difference of nature between free regimes and those that are not and makes it possible to take the Roman republic or the Athenian democracy as models.

KEYWORDS : Hobbes, republicanism, mixed government, sovereignty, citizen.

Revue de synthèse : $4^{e} \mathrm{~S} . \mathrm{N}^{\mathrm{os}}$ 2-3, avril-septembre 1997, p. 221-236. 
ZuSAMMENFASSUNG : Hobbes kritisiert die Theorie der gemischten Regierungsform, ohne die gemischte Regierungsform als republikanisch zu bezeichnen. Die Kritik des Republikanismus ist bei ihm Kritik an der Definition der Freiheit als Teilnahme der Bürger an Entscheidungsprozessen über Angelegenheiten, die die Gemeinschaft betreffen. Die Argumentation dieser Kritik ist im Wesentlichen in den Werken Elemente des natürlichen und politischen Gesetzes und De cive zu finden. Der Leviathan faßt sie zusammen und erhärtet sie. Gleichzeitig wird die Idee der Wesensgleichheit der höchsten Gewalt in allen politischen Gesellschaftsformen auf ganz neue Weise entwickelt. Durch diese Idee wird also die republikanische Behauptung, nach der die freien und unfreien Regierungsformen wesensverschieden seien, widerlegt. Daraus folgt, daß.s die römische Republik oder die Demokratie von Athen als Vorbild erklärt werden kann.

STiCHWÖRTER: Hobbes, Republikanismus, gemischte Regierungsform, höchste Gewalt, Bürger.

Jean TERREL, né en 1943, ancien élève de l'École normale supérieure, est actuellement professeur à l'université Michel de Montaigne à Bordeaux. Ses recherches portent sur l'histoire de la philosophie politique à l'âge classique. Il est l'auteur de Hobbes, matérialisme et politique (Paris, Vrin, 1994).

Adresse : UFR de philosophie, Université Bordeaux III Michel de Montaigne, Domaine universitaire, 33405 Talence Cedex. 
Dans sa philosophie politique (1640-1651), et ensuite dans son histoire de la guerre civile (1668) ${ }^{1}$, Thomas Hobbes critique les principaux aspects de la tradition républicaine : l'admiration pour les républiques antiques, la description de la citoyenneté comme forme de réalisation de l'excellence humaine, l'éloge du gouvernement mixte. Pour comprendre cette critique, il faut déterminer le rôle de cette tradition dans la vie politique anglaise vers 1640 : il s'agit d'interpréter de façon nouvelle, à travers la rhétorique du gouvernement mixte, les institutions politiques anglaises qui associent le Parlement à l'exercice de l'autorité royale. Il faut ensuite analyser le contenu de la critique. Hobbes examine la théorie du gouvernement mixte et son application à l'Angleterre, mais cet examen ne met pas en évidence ce qui différencie l'interprétation traditionnelle de la constitution anglaise et la nouvelle lecture qui en est faite en termes de gouvernement mixte. Dans les Éléments de la loi naturelle et politique (1640) et dans le De cive (1642), le rejet explicite du républicanisme prend la forme d'une dévalorisation du désir de citoyenneté et d'un refus de la définition républicaine de la liberté comme participation à la délibération sur les affaires communes. Pour des raisons internes au développement de la théorie, et aussi pour des raisons de conjoncture, le Leviathan (1651) résume et durcit la critique du républicanisme jusqu'à donner une impression de sectarisme, tout en développant de façon nouvelle une idée présente dès les premières œuvres, celle d'une identité d'essence de la souveraineté dans toutes les sociétés civiles. Cette idée contredit la thèse républicaine selon laquelle il y aurait une différence de nature entre les régimes libres et ceux qui ne le sont pas, mais elle permet en même temps de constituer en modèle la république romaine ou la démocratie athénienne.

I. - LA CRITIQUE DU GOUVERNEMENT MIXTE

Si l'on suit les analyses de John Greville Agard Pocock ${ }^{2}$, la tradition républicaine s'implante durablement en Angleterre vers 1640, au

1. Behemoth, éd. Ferdinand Tonnies, $2^{\mathrm{e}}$ éd. Londres, Frank Cass, 1969 (cité par la suite comme TonnIEs), trad. franç. Luc Borot, Paris, Vrin, 1990 (cité par la suite comme Vrin).

2. Voir, par ex., The Ancient Constitution and the feudal law. A reissue with a retrospect, Cambridge, Cambridge University Press, 1987, p. 310 sq. 
moment où la crise politique met en question le langage de l'ancienne constitution précédemment utilisé pour décrire le rôle respectif du roi et du Parlement. Selon ce langage, l'ancienne constitution du royaume associe sans les mêler deux pouvoirs, le pouvoir politique exercé conjointement par le roi et le Parlement, et le pouvoir régalien qui permet au roi de décider seul quand les intérêts supérieurs du royaume paraissent en jeu. Le pouvoir régalien manifeste ce qui, dans l'autorité du roi, est d'origine divine. Le pouvoir politique est susceptible d'interprétations opposées, selon la manière d'apprécier le rôle respectif du roi et du Parlement.

Selon le langage républicain, le gouvernement mixte mêle la monarchie, l'aristocratie et la démocratie. En Angleterre, l'histoire a réalisé un heureux mélange, un gouvernement associant les trois états du royaume, le roi, les Lords et les Communes. Grâce à une double innovation - les évêques ne sont plus l'un des états constitutifs du royaume, le roi devient l'un de ces états - le royaume est libéré de la tutelle des clercs et le pouvoir royal perd sa transcendance : la légitimité des institutions ne tient plus au droit divin mais à l'autorité de la tradition.

Le document qui interprète le plus clairement les institutions anglaises de cette manière est la Réponse de Sa Majesté aux dix-neuf propositions des deux chambres du Parlement ${ }^{3}$. À cette date (1642), les deux premières œuvres politiques de Hobbes, les Éléments de la loi naturelle et politique et le $D e$ cive $e^{4}$, sont rédigées. En réalité, la lecture républicaine de la tradition anglaise est bien antérieure : elle apparaît dès le milieu du xvi ${ }^{e}$ siècle et s'accompagne d'une énumération nouvelle des trois états : le roi, les Lords, les Communes, et non plus ceux qui prient, ceux qui combattent et ceux qui travaillent. À la charnière du $x v I^{e}$ et du $x{ }^{e}{ }^{e}$ siècle, cette théorie révisée est utilisée par les presbytériens contre les évêques et contre le roi lorsqu'il revendique une autorité absolue. De ce fait, la théorie devient subversive, elle est une " position dangereuse ${ }^{5}$ " réprimée par les autorités. Le thème réapparaît à partir de 1630 et se répand entre 1640 et 1642 , à un moment où les modérés du camp parlementaire et les royalistes modérés, qui feront la guerre civile au côté du roi, font bloc contre la politique absolutiste et cléricale de Charles $I^{\text {er }}$. En 1642, l'alliance anti-absolutiste se défait parce que la majorité parlementaire revendique le droit de contrôler l'exécutif. Deux

3. Sur ce point, voir John Greville Agard Pocock, The Machiavellian Moment, Princeton, Princeton University Press, 1975, p. 361-366, qui cite de très larges extraits de la Réponse de Sa Majesté que je commente ici.

4. Elements of law natural and politic, éd. F. Tonnies, $2^{\mathfrak{e}}$ éd. Londres, Frank Cass, 1969 (cité par la suite comme Elements). De cive, éd. F. TonniEs, Oxford, Clarendon Press ( $«$ The Clarendon Edition of the philosophical works of Thomas Hobbes ", t. II), 1983 (cite par la suite comme De cive).

5. Pour un examen détaillé de cette question, voir Michael Mendle, Dangerous Positions, Tuscaloosa, The University of Alabama Press, 1985. 
royalistes modérés, Lucius Cary Falkland et John Colepeper, rédigent la réponse du roi aux revendications du Parlement : attribuer aux Communes le droit de contrôler la nomination des conseillers royaux serait détruire le fragile équilibre entre les trois états au profit de l'élément populaire. Ce qui était au départ subversif est devenu l'argument de certains défenseurs du roi contre le Parlement accusé de prétendre à la suprématie.

Quand il critique la théorie du gouvernement mixte, Hobbes pense de plus en plus clairement à la situation anglaise. En 1640, il prend comme exemple de cette théorie la séparation de trois pouvoirs : « pouvoir de faire les lois donné à quelque grande assemblée démocratique », " pouvoir de juger attribué à quelque autre assemblée », " pouvoir d'administrer les lois confié à une troisième assemblée ou à un seul homme " ${ }^{6}$. En 1642, nous nous rapprochons de la situation anglaise. L'exemple est celui d'une monarchie mixte : "nomination des magistrats et décision de paix et de guerre aux mains du roi », "affaires judiciaires aux mains des grands », " vote des impôts aux mains du peuple », «puissance de faire les lois aux mains de tous ensemble ${ }^{7}$. Enfin, en 1651, nous rejoignons la version anglaise du régime mixte : le pouvoir de lever les impôts dépend d'une assemblée générale (Hobbes pense manifestement aux Communes), le pouvoir de conduire et de commander dépend d'un seul homme, «le pouvoir de faire les lois [...] dépend de l'accord accidentel, non seulement de ces deux parties, mais encore d'une troisième ${ }^{8} »$. Plus haut, Hobbes a noté que l'opinion selon laquelle les pouvoirs « sont divisés entre le roi, les Lords et la chambre des Communes " était reçue par le plus grand nombre en Angleterre, ce qui a provoqué la guerre civile ${ }^{9}$. Dans Behemoth, l'amour pour la monarchie mixte est attribué à « la majeure partie des Lords du Parlement et de la gentry dans toute l'Angleterre ${ }^{10}$ " et aux conseillers du roi ", ce qui est une allusion évidente à la réponse rédigée par Falkland et Colepeper.

Dans les premières œuvres, la présentation de la doctrine du régime mixte n'élimine pas toute trace de rhétorique républicaine. Des partisans d'un tel régime, il est dit qu'ils veulent éviter l'esclavage ${ }^{12}$. L'opposition des pouvoirs despotique et politique est un thème républicain. Plus intéres-

6. Elements, part. II, chap. I, $\S 15$.

7. De cive, chap. vil, $\$ 4$.

8. Leviathan, chap. xxix, éd. Crawford Brough Macpherson, Harmondsworth, Middx, Penguin Books, 1968 (cité par la suite comme MCPH), p. 372, trad. François TrICAud, Paris, Sirey, 1971, (cité par la suite comme TR), p. 352.

9. Leviathan, chap. xVII, MCPH, p. 236-237, TR, p. 188.

10. Behemoth, Dialogue I, TonNIEs, p. 33, Vrin, p. 72.

11. Behemoth, Dialogue III, TonNIEs, p. 116-117, Vrin, p. 157.

12. Elements, part. II, chap. I, § 15; De cive, chap. vil, \$4. 
sant encore, dans les Elements, Hobbes écrit que « l'erreur sur le gouvernement mixte vient de ce qu'on n'a pas compris ce que signifie l'expression corps politique et qu'elle signifie non pas la concorde mais l'union de plusieurs hommes ${ }^{13}$ ". La concorde est la convergence provisoire et fragile des volontés, alors que l'union suppose l'existence d'une souveraineté non divisée. Hobbes reproche à Aristote de concevoir la cité selon le modèle de la concorde ${ }^{14}$. Puisque Aristote est une des autorités de la tradition républicaine, nous pouvons être tenté de reconstruire entre Hobbes et cette tradition une opposition frontale dont l'enjeu serait la souveraineté, reconnue par l'un et ignorée par les autres à travers la défense du régime mixte. En réalité, le texte des Elements qui vient d'être cité vise en premier lieu une tradition juridique antérieure à l'humanisme civique. Si l'on a manqué l'union de la cité en la confondant avec un simple consensus, c'est que l'on n'a pas remarqué que la cité était, comme les corporations, une personne unique. La critique atteint aussi Aristote, moins sans doute l'Aristote spécifiquement républicain que celui qui alimente depuis saint Thomas d'Aquin la pensée des théologiens et des juristes : les références à ce qui oppose pouvoirs politique et despotique, ou encore au régime mixte sont, de ce point de vue, bien antérieures à la naissance de l'humanisme civique dans 1'Italie de la Renaissance ${ }^{15}$.

Hobbes n'établit aucun lien entre la doctrine favorable au gouvernement mixte et l'admiration excessive pour l'Antiquité. Quand il dresse la liste des Anciens (Platon, Aristote, Cicéron, Sénèque, Plutarque) qui ont une mauvaise influence sur les Modernes, Polybe, l'autorité par excellence en matière de gouvernement mixte, n'est pas cité. L'erreur sur la divisibilité de la souveraineté est expliquée par l'influence de " quelques hommes qui font profession de science juridique ${ }^{16} \gg$. Elle n'est pas pour Hobbes une nouveauté républicaine mais un préjugé enraciné dans la tradition juridique anglaise. Cette opinion des juristes est rapprochée de celle des théologiens qui veulent confier le pouvoir temporel au souverain civil et le pouvoir spirituel aux évêques ou aux Églises ${ }^{17}$. Or l'interprétation républicaine de la constitution anglaise avait précisément pour effet de refuser que les évêques revendiquent, au nom de leur autorité spirituelle, une fonction politique spécifique. Dans le Leviathan, l'erreur sur le régime mixte est

13. Elements, part. II, chap. VIII, § 7.

14. De cive, chap. v, $\$ 5$. Dans le texte équivalent des Elements, Hobbes se contente d'opposer la concorde des abeilles dans la ruche et l'union des hommes dans la cité, sans reprocher à Aristote d'avoir compté les abeilles au rang des animaux politiques (part. I, chap. XIX, \& 5).

15. Sur l'analyse faite par saint Thomas d'Aquin du régime mixte, voir Somme théologique, 1267-1273, Paris, Cerf, 1984, t. II, section I, partie I, Question 95, a. 4, réponse, p. 602 et ibid., section I, partie II, Question 105, a. 1, réponse, p. 701-702.

16. Leviathan, chap. XXIX, MCPH, p. 368, TR, p. 347.

17. De cive, chap. $\mathrm{XII}, \S 5$; Leviathan, chap. $\mathrm{xxIx}$, МсPH, p. 370-372, TR, p. 349-352. 
aussi rattachée à la maladresse des premiers constructeurs de la monarchie anglaise qui se sont contentés « de moins de pouvoir qu'il n'est nécessairement requis pour la paix et la défense de la République ". La même faute avait été commise à Rome où «ni le peuple ni le Sénat ne revendiquaient l'intégralité du pouvoir ${ }^{18} »$. Il ne s'agit pas de rattacher l'erreur anglaise à la mauvaise influence des républicains romains, mais de montrer que l'ignorance de l'essence de la souveraineté entraîne, à des siècles de distance, des maladresses comparables.

$\mathrm{Du}$ point de vue de la doctrine absolutiste, la différence entre les langages qui se réfèrent respectivement à la tradition juridique anglaise et au gouvernement mixte s'estompe, ce qui scandalise Edward, comte de Clarendon. Pour ce dernier,

«l'idée selon laquelle le pouvoir est divisé entre le Roi, les Lords et la Chambre des Communes [...] est une opinion dont on n'avait jamais entendu parler en Angleterre avant le début de la Rébellion et à laquelle toutes les Lois d'Angleterre s'opposent de la façon la plus claire $[\ldots]^{19} \mathrm{~m}$.

L'analyse de Hobbes procède de « son ignorance extraordinaire et notoire pour les Lois et la constitution du gouvernement de l'Angleterre ${ }^{20} »$.

\section{II. - LA CRITIQUe DE L'ASPIRATION À LA CITOYENNETÉ DANS LES ELEMENTS ET LE DE CIVE}

Il existe un trait constant dans l'attitude de Hobbes envers le républicanisme : de 1640 à 1668, des Elements à Behemoth, il considère que l'admiration excessive pour les républiques antiques dispose les hommes à la guerre civile. Lorsqu'il dresse la liste des opinions séditieuses, les doctrines qui proviennent de cette admiration et l'opinion favorable au gouvernement mixte occupent des places distinctes. En 1640 et 1642, la critique se développe également dans un autre contexte : il s'agit de souligner les particularités de la démocratie et de montrer qu'elles ne justifient pas la préférence pour l'État populaire ou l'hostilité à la monarchie. À première anályse, cela tendrait à relativiser la critique. Si le gouvernement mixte est récusé pour des raisons de principe, au nom de l'essence indivisible de la souveraineté, la démocratie est parfois acceptable, puisque l'on doit obéissance au régime établi dès l'instant où il assure la sécurité. La science politique

18. Leviathan, chap. xxIx, MCPH, p. 364-365, TR, p. 343-344.

19. "A survey of Mr Hobbes his Leviathan ", in Leviathan, contemporary responses to the political theory of Thomas Hobbes, Bristol, Thoemmes Press, 1995, p. 213.

20. Ibid., p. 214. 
démontre que le souverain doit posséder une autorité illimitée, le choix du souverain est une affaire de prudence pratique.

Certains hommes désirent avoir part aux charges publiques. Ce sont d'abord, selon les Elements, ceux qui ont institué une république sans y être contraints par la supériorité d'un vainqueur :

« [...] celui qui s'est assujetti de lui-même, sans y être contraint, voit là une raison pour être mieux traité que celui qui le fait sous la contrainte et, venant à la sujétion librement [freely], il se dénomme lui-même homme libre [freeman], quoiqu'il soit sujet; par là il apparaît que la liberté [liberty] n'est pas le fait d'être exempt de la sujétion et de l'obéissance au pouvoir souverain, mais un état où l'on peut espérer mieux que ceux qui ont été assujettis par force et conquête [...] Donc la liberté [freedom] dans les républiques n'est rien d'autre que l'honneur d'une égalité de faveur avec d'autres sujets, et la servitude est l'état du reste. Un homme libre [freeman] peut donc attendre des emplois honorables, mieux qu'un serviteur. Et c'est tout ce qu'on peut entendre par liberté [liberty] du sujet. Car, dans tous les autres sens, la liberté est l'état de celui qui n'est pas sujet ${ }^{2 !}$.»

Ce texte distingue les États institués librement et les États qui résultent de la soumission à un conquérant. Existerait-il, comme le disent les républicains, une différence de nature entre les régimes libres et les régimes despotiques? La réponse de Hobbes est négative : en tout régime la sujétion est la même, vous devez obéir au souverain tant qu'il assure votre sécurité. Pourtant - et c'est une relative concession à l'idéologie républicaine - il est légitime de distinguer deux types de sujets, et on peut accepter de parler à leur propos de liberté [liberty ou freedom] et de servitude : de liberty pour désigner l'état de celui qui s'est soumis librement et peut raisonnablement espérer accéder aux charges publiques, et de freedom pour désigner l'honneur de partager ces charges avec d'autres citoyens.

Cette analyse est propre aux Elements. Il existe une seconde raison, exposée aussi dans le $\mathrm{De}$ cive ${ }^{22}$, pour désirer la liberté civile. Certains hommes vivent à leur aise, à l'abri de la pauvreté et de la violence : dans la bataille de leurs appétits, la voie est libre pour le désir de gloire qu'avive la lecture des philosophes et historiens de l'Antiquité. Si le souverain ne leur confie pas les honneurs auxquels ils aspirent, ils se croient traités en esclaves.

Si nous en restons aux intentions, ces hommes aspirent seulement à une charge honorifique. Sauf à vivre en démocratie, ils ne peuvent être tous satisfaits. Si maintenant nous ne considérons plus les intentions mais le sens des mots, ils demandent la souveraineté. En effet, puisque « la liberté

21. Elements, part. II, chap. Iv, $\$ 9$.

22. Elements, part. II, chap. VIII, \& 3; De cive, chap. XII, §10. 
ne peut coexister avec la sujétion ", seul le souverain est libre. S'il comprend ce qu'il dit, celui qui demande la liberté demande le commandement suprême. S'il vit dans une monarchie, il veut « soit avoir la souveraineté à son tour, soit être le collègue de celui qui l'a, soit changer la monarchie en démocratie ${ }^{23}$ ". À défaut de pouvoir dominer seul ou à quelques-uns, on veut gouverner en commun, on veut la démocratie. "Comme Aristote le dit fort bien, "Le fondement ou l'intention d'une démocratie est la liberté [...] Car les hommes d'ordinaire disent qu'ils ne peuvent avoir part à la liberté que dans une république populaire" 24 ". Par rapport au texte précédent, la critique de l'idéal civique se précise. Hobbes n'accepte plus de nommer liberté l'exercice d'une charge concédée par le souverain. Dans le cas d'une souveraineté collective, on ne pourra parler de liberté à propos des citoyens qui ont part à l'assemblée souveraine : comme individus, ils restent des sujets. Dans le texte équivalent du De cive, Hobbes reproche à Aristote d'avoir parlé, à propos de la démocratie, de liberté et non de souveraineté, et il élimine de sa traduction l'idée que le citoyen a part à la liberté commune ${ }^{25}$.

Si l'on admet que les citoyens en démocratie ont part à l'imperium, ce régime a au moins l'avantage d'offrir un débouché plus large au désir de gloire. Devant cet ultime argument, les Elements restaient silencieux. Au contraire, le De cive développe longuement la thèse républicaine :

«Mais peut-être que quelqu'un dira que l'État populaire est de beaucoup préférable au monarchique, car, là où tous se mêlent des affaires publiques, on donne à chacun le pouvoir de montrer en public sa prudence, son savoir et son éloquence, dans les délibérations à propos des choses les plus difficiles et les plus importantes, ce qui, à cause du désir inné de louange propre à la nature humaine, est la plus agréable de toutes les choses pour tous ceux qui possèdent ou croient posséder ces qualités mieux que tous les autres ${ }^{26}$.»

23. Elements, part. II, chap. viI, § 3 .

24. Elements, ibid. C'est dans ce texte des Elements que se trouve la traduction la plus

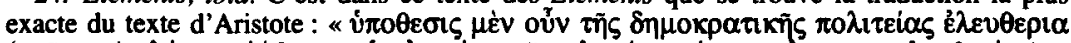
( Voir ArISTote, Politique, texte établi et traduit par Jean Aubonnet, 5 vol., Paris, BellesLettres, 1960-1989 (cité par la suite comme Politique), liv. VI, chap. II.

25. Voir De cive, chap. $x, \S 8:$ : Ce qui en impose est la participation égale aux charges publiques et au commandement [imperii]. Car là où existe le Commandement [imperium] du peuple, chaque citoyen participe au commandement en tant que partie du peuple qui commande. Tous participent également aux charges publiques pour autant qu'ils ont des suffrages égaux pour élire les magistrats et les ministres publics. Et c'est ce qu'Aristote a voulu dire, parlant lui aussi selon la coutume du temps, de liberté là où il s'agit de commandement : (Politique, liv. VI, chap. II) "Dans l'État populaire la liberté existe à partir du principe de base de ce régime [latin, libertas est ex suppositione; anglais, there is liberty by supposition\}. C'est ce que dit le vulgaire, comme si personne n'était libre en dehors de ce régime." "

26. De cive, chap. $\mathrm{x}, \& 9$. 
Pour Hobbes, la démocratie antique n'a pas pour principe le sacrifice de l'égoïsme individuel au bien public, ce que Montesquieu et Rousseau nommeront vertu, et qui est chrétien plus que paien, mais l'honneur. Comment un principe aristocratique peut-il être le ressort de la démocratie? Selon Hobbes, le désir de gloire est présent en tout homme et devient dominant quand il n'est pas combattu par des soucis matériels plus pressants. En ce sens, il caractérise les puissants. Si la démocratie donne à chacun le pouvoir [potestas] de faire montre de sa prudence, de son savoir et de son éloquence, ce pouvoir est de fait utilisé par une aristocratie d'orateurs. C'est donc d'abord au désir de gloire des puissants que la monarchie fait obstacle. Hobbes ne critique pas ce désir au nom des valeurs chrétiennes, mais au nom de l'intérêt bien compris de l'individu : blessures d'amour-propre, développement de la haine, négligence de nos affaires domestiques, obligation de découvrir ce que nous avons dans l'âme ${ }^{27}$. L'exemple de Coriolan, au paragraphe quinze, est significatif. Coriolan n'est pas un orateur démocrate. Son excellence est militaire. En ce qui concerne le désir de gloire, il est pour Plutarque une exception: "Pour les autres, le but de la valeur [était la gloire], pour lui le but de la gloire était la joie de sa mère ${ }^{28}$. » Pour Hobbes :

«Il n'y a aucune raison pour laquelle n'importe qui ne préférerait pas vaquer à ses affaires privées plutôt qu'aux affaires publiques, si ce n'est qu'on y trouve l'occasion de montrer son éloquence, d'acquérir ainsi réputation d'intelligence et de prudence et, de retour à la maison, de se vanter auprès des amis, parents et femme de ses belles actions. "

Ce qui était l'exception chez les Anciens, la transformation de la gloire en moyen au service de fins privées, est devenue la règle chez les Modernes. Même pour un ambitieux, l'espace privé vaut davantage que l'espace public.

Comment expliquer que Hobbes disjoigne ainsi la critique du gouvernement mixte et la critique de l'idéal de citoyenneté ? On ne peut simplement invoquer la rigueur qui le contraint à distinguer ce qui relève de l'universel - l'indivisibilité de la souveraineté — et ce qui relève des contingences historiques - selon que le souverain est un homme ou une assemblée, la république risque d'être gouvernée de façon différente. Car la question de la citoyenneté n'est pas seulement discutée au moment où l'on examine les

27. De cive, chap. $x, \S 9$ : la participation à la délibération publique nous contraint «à dévoiler sans profit à tous nos avis et souhaits dans des domaines où cela n'est pas nécessaire ".

28. Vies, Paris, Belles lettres, 1964, t. III, p. 179. 
particularités de la démocratie, elle l'est aussi quand sont réfutées les opinions subversives : examinées dans le même contexte, les questions du gouvernement mixte et de la liberté républicaine font alors l'objet d'un traitement distinct. Pour comprendre l'attitude de Hobbes, il faut analyser comment les deux thèmes, tous deux constitutifs de la tradition républicaine, dépendent l'un de l'autre ou sont autonomes. Chez Aristote, Platon ${ }^{29}$ ou Machiavel, ils sont indissociables : le régime mixte est le moyen de faire participer tous les citoyens à la délibération publique, en tenant compte du fait qu'ils ne sont pas égaux en tout. Il a aussi l'avantage d'éviter qu'une fraction de la cité, même majoritaire, confisque le pouvoir et exerce une domination despotique sur les autres citoyens. Chez Polybe, le régime mixte est pensé sans relation forte avec l'exigence de citoyenneté. Dans la tradition juridique antérieure à l'humanisme civique, il s'agit davantage de freiner le pouvoir royal que de permettre à chaque sujet de se réaliser comme animal politique. Bodin lui-même critique de façon indépendante la théorie polybienne du gouvernement mixte et la définition aristotélicienne du citoyen. Dans le Methodus ${ }^{30}$, il va jusqu'à considérer qu'Aristote est un adversaire du régime mixte! Hobbes fait un pas de plus : il ne se contente pas de séparer les deux questions, il traite la première en négligeant la rhétorique républicaine. C'est peut-être aller à l'essentiel. Il n'est pas sûr que les défenseurs anglais du gouvernement mixte aient été, en 1642, de vrais républicains : le gouvernement mixte est-il encore républicain s'il n'est plus le régime qui met le pouvoir au milieu des citoyens de manière à ce qu'il ne puisse être confisqué par personne?

\section{III. - LA CRITIQUE DU RÉPUBLICANISME DANS LE LEVIATHAN}

Du De cive au Leviathan, la critique du désir de liberté civique semble s'être considérablement appauvrie. Sauf de façon fugitive ${ }^{31}$, Hobbes ne

29. Les Lois, Paris, Gallimard, 1950, liv. IV, 712 c sq.

30. Voir Methodus ad facilem historiarum cognitionem, chap. vl, in Euvres philosophiques de Jean Bodin, texte établi, traduit et publié par Pierre Mesnard, Paris, Presses universitaires de France, 1951, p. 366 : «Et, en ce qui concerne Sparte, Aristote leur a donné l'occasion de se tromper en y reconnaissant un gouvernement mixte, en rapportant sans la réfuter l'opinion de certains auteurs qui la disent en partie populaire, en partie monarchique, en partie aristocratique. Mais, comme en dehors de cette controverse, il n'a reconnu que trois modes de gouvernement, il ne semble pas utile de chercher plus loin son avis, alors qu'il est si manifeste dans ses cuvres. »

31. Voir Leviathan, chap. XVIII, MCPH, p. 231, TR, p. 182 : Hobbes parle de «l'ambition de quelques-uns, qui sont plus favorables au gouvernement d'une assemblée, dont ils peuvent nourrir l'espoir de faire partie, qu'à celui d'une monarchie, pouvoir dont ils n'ont pas l'espoir de jouir ». 
mentionne plus le désir de participer à la vie publique et n'examine plus les causes d'un tel désir. Il faut vivre en monarchie et avoir une connaissance purement livresque de la démocratie pour imaginer que « les sujets d'une République populaire jouissent de la liberté, alors que dans une monarchie tous seraient esclaves ${ }^{32}$ ». Ceux qui vivent sous un gouvernement populaire ne découvrent rien de tel dans leur expérience. Tout au plus adhèrent-ils à la propagande officielle. Ainsi enseignait-on aux Athéniens, «pour les détourner du désir de changer de régime [...] qu'ils étaient des hommes libres ${ }^{33} »$. En l'absence de science politique, on ne pouvait enseigner impartialement aux hommes qu'ils doivent obéir au souverain de leur république. On cherchait donc à obtenir l'obéissance en diffusant des opinions partisanes. Aristote et Cicéron ont eu le tort de transformer ces opinions en doctrine politique. Aujourd'hui où ces auteurs sont devenus de véritables autorités en matière politique, leurs auvres sont des poisons pour " les jeunes gens, et en général tous ceux auxquels fait défaut l'antidote d'une solide raison ${ }^{34} "$.

Comment expliquer l'abandon de la critique argumentée au profit d'un apparent sectarisme? Dans les années 1640-1642, Hobbes insiste sur les particularités des républiques populaires : «La puissance est égale en toute espèce de cité ", mais, selon que le souverain est un monarque ou une assemblée populaire, il est probable que la cité sera administrée ou gouvernée de façon différente. C'est encore se placer sur le terrain des républicains, en leur montrant que l'examen des particularités de l'État populaire met en question leur préférence partisane. Hobbes choisit cette manière d'argumenter parce qu'il juge que l'aspiration à la citoyenneté joue un rôle important dans les troubles qui préparent la guerre civile. Au moment où le Leviathan est rédigé et publié, la guerre civile est terminée. Selon le jugement de Hobbes, il existe en Angleterre un pouvoir de fait, capable d'assurer la sécurité que tout individu recherche quand il consent à devenir membre d'une république. Il n'est plus temps de discuter des particularités de tel ou tel régime, mais de défendre le temple de la souveraineté sans considérer son ou ses occupants. Il faut suivre l'exemple de « ces simples et impartiales créatures qui, au Capitole de Rome, protégèrent par leur tapage ceux qui se trouvaient dedans, non parce que c'était eux, mais parce qu'ils étaient là [not because they were they, but there] ${ }^{35} "$. En 1651, on pratique la même impartialité en consentant à obéir au régime sans considérer que ses fondateurs ont violé la loi naturelle par la rébellion.

32. Leviathan, chap. XxIx, MCPH, p. 369 , TR, p. 349.

33. Leviathan, chap. XXI, MCPH, p. 267, TR, p. 228.

34. Leviathan, chap. XXIX, MCPH, p. 369, TR, p. 348.

35. Leviathan, dédicace, trad. F. Tricaud légèrement modifiée, McPH, p. 75, TR, p. 1. 
L'insistance nouvelle sur la thèse selon laquelle le pouvoir souverain est le même dans toutes les républiques a donc pour premier effet l'abandon de l'examen détaillé des particularités des États populaires. On aurait tort cependant de s'arrêter à l'apparence d'un durcissement de la critique, car le nouveau point de vue permet aussi de mettre en évidence des traits communs aux républiques antiques et aux monarchies modernes. Les républiques antiques ont l'intérêt de rendre plus visibles certains caractères essentiels de toute autorité souveraine. Considérons trois opinions que l'on oppose couramment à l'absolutisme: l'institution du souverain résulte d'une convention passée entre lui et ses sujets; l'ensemble des citoyens est supérieur au souverain; il existe à côté du souverain une représentation du peuple distincte de lui. Dans l'hypothèse de la démocratie, l'absurdité de ces opinions saute aux yeux. Les Romains n'ont pas passé une convention avec le peuple romain, ils n'ont pas promis d'obéir à telle ou telle condition $^{36}$. L'ensemble des citoyens n'est pas supérieur au peuple souverain, c'est-à-dire à lui-même ${ }^{37}$. Enfin, le peuple souverain n'a nul besoin de susciter une représentation de lui-même distincte de lui ${ }^{38}$. Si vous êtes impartial, vous devez accepter qu'un roi dispose de la même souveraineté : que son autorité ne soit limitée par aucune condition contractuelle, qu'il ne soit pas inférieur à la personne formée par ses sujets puisqu'il incarne cette personne, enfin qu'il soit l'unique représentant absolu du peuple puisqu'il représente chaque sujet et porte la personne qu'ils forment à eux tous. Une conclusion étrange s'en dégage : si les admirateurs de l'Antiquité étaient conséquents, ils aboutiraient à la nécessité de l'absolutisme. Au chapitre XXI consacré à la liberté des sujets, Hobbes ajoute deux arguments qui vont dans le même sens. Bien comprise, la liberté vantée par les républicains est la souveraineté dont disposait chaque cité pour assurer sa sécurité et celle de ses membres, elle doit à l'évidence être accordée, sauf partialité, aux monarchies ${ }^{39}$. De même, chaque république dispose du droit de répri-

36. Voir Leviathan, chap. xVII, MCPH, p. 231, TR, p. 182 : « [...] nul n'est assez sot pour dire, par exemple, que le peuple de Rome avait fait une convention avec les Romains, comme quoi il détiendrait la souveraineté sous telle ou telle condition, les Romains pouvant légitimement déposer le peuple romain au cas où celles-ci ne seraient pas remplies. »

37. Hoвses, in Leviathan, chap. XVII, MCPH, p. 237, TR, p. 190, critique la formule selon laquelle « le roi est major singulis et universis minor », plus puissant que ses sujets pris un à un, et moins puissant que l'ensemble. Selon ArIstote, in Politique, liv. III, chap. xv, 1286 b 28-b 38, cette formule convient pour un roi qui gouverne selon la loi et non selon son bon vouloir : un tel roi doit avoir une force armée suffisante pour que la loi soit obéie, il doit être moins puissant que la multitude pour n'être pas tenté de violer la légalité. R. Hooker considère que la formule est conforme aux institutions anglaises : voir Richard Hooker, of the laws of ecclesiastical polity, liv. VIII, chap. II, \$ 2, Cambridge, Cambridge University Press, 1989, p. 143.

38. Leviathan, chap. XIx, MCPH, p. 240, TR, p. 193.

39. Leviathan, МсPH, p. 266, TR, p. 227. 
mer l'un de ses sujets innocents ${ }^{40}$. On comprend généralement que le souverain, dans une telle hypothèse, viole la loi naturelle sans en être comptable devant les hommes : David n'a pas violé les droits d'Urie en l'envoyant à la mort pour épouser Bethsabée, il a péché contre Dieu. Mais Hobbes enchaîne sur l'ostracisme pratiqué par Athènes. Contrairement à ce que croit comprendre Samuel Sorbière quand le même exemple est examiné dans le De cive $^{41}$, la pratique de l'ostracisme ne contredit pas nécessairement la loi de nature, même si les citoyens bannis sont innocents. Il est parfois nécessaire à la sécurité collective de frapper un innocent, et c'est au souverain de juger de cette nécessité. Cela ne signifie pas que la politique est immorale, mais plutôt que la morale politique n'est pas exactement celle qui vaut pour l'individu. De ce point de vue, Hobbes rejoint Aristote qui explique que l'ostracisme n'est pas une mesure spécifique aux régimes déviés et qu'il peut avoir sa place dans une $\pi 0 \lambda \imath \tau \varepsilon i ́ \alpha$ (constitution) bien constituée ${ }^{42}$.

Une telle lecture de l'expérience des républiques antiques est à l'opposé de celle des républicains humanistes. Pour éclairer leur combat présent, ces derniers cultivent le souvenir d'une expérience particulière, le moment rare où une communauté de citoyens a opposé sa souveraineté aux assauts de la fortune. Selon Hobbes, cela revient à se perdre dans la particularité des événements, comme les grammairiens qui « décrivent les règles du langage d'après l'usage de leur époque, ou les règles de la poésie d'après les poèmes d'Homère et de Virgile ". Ce qui est propre aux républiques antiques est impropre à servir de fondement à la science politique ${ }^{43}$. Pour être impartiale, la science politique doit écarter le particulier et retenir l'universel. Ce geste est une prise de parti : Hobbes met dans la cité grecque et dans la république romaine ce qu'il a besoin d'y trouver, un souverain absolu $^{44}$, ce qui lui permet d'opposer sa propre impartialité aux préférences partisanes des républicains. Il faut reconnaître que le concept de souverai-

40. Leviathan, MCPH, p. 264-266, TR, p. 224-226.

41. «Pares in hoc Democratia et Monarchia [...]» : «En cela [la répression contre un citoyen trop puissant] la démocratie et la monarchie sont identiques [...] », ce que Samuel SoRBIÉRE traduit : «La démocratie et la royauté ne sont pas moins coupables [...] », chap. $x, \S 7$.

42. Politique, liv. III, chap. xiI, 1284 a 18-b 18. Aristote va jusqu'à dire « qu'on ne doit pas tenir pour absolument juste la position des adversaires de la tyrannie et du conseil que Périandre donna à Thrasybule ": couper les épis qui dépassaient pour égaliser le champ.

43. Voir Leviathan, chap. Xxa, McPH, p. 267, TR, p. 228 : "Dans nos pays occidentaux, nous sommes accoutumés à recevoir nos opinions touchant l'institution et les droits des républiques d'Aristote, de Cicéron et d'autres Grecs et Romains, qui, vivant sous des États populaires, ne déduisirent pas ces droits des principes de la nature, mais les transcrivirent dans leurs ouvrages conformément à ce qui se faisait dans leurs propres républiques, qui étaient populaires. "

44. Benjamin Constant s'accorde avec Hobbes pour mettre en évidence les tendances absolutistes des démocraties antiques. Constant se réfere à plusieurs reprises à l'ostracisme. Voir «De la liberté des Anciens comparée à celle des Modernes», in De la liberté chez les Modernes, écrits politiques de B. Constant, textes choisis, présentés et annotés par Marcel GAUCHET, Paris, Librairie générale française, 1980. 
neté n'est théorisé ni dans la Politique, ni dans la tradition républicaine. Il faut cependant se demander ce qui rend possible cette interprétation de l'expérience antique dans le langage de la souveraineté. Aristote est au plus près de l'expérience politique grecque lorsqu'il considère que la $\pi 0 \lambda \imath \tau \varepsilon i \alpha$ (constitution) est la forme de la cité, son principe d'unité et d'identité ${ }^{45}$ : pas de $\pi$ ó $\lambda ı \varsigma$ (cité) sans $\pi 0 \lambda ı \imath \varepsilon i \alpha$ (constitution). Hobbes substitue le souverain à la constitution comme forme de la république. La différence est considérable, mais un trait essentiel subsiste, l'idée que la communauté, à travers ce qui l'institue, $\pi$ o $\lambda$ i $\tau \varepsilon i \alpha$ (constitution) ou souverain, exerce un pouvoir qui ne connaît pas de limites morales externes au champ politique. Il importe que ce pouvoir se donne des limites pour se protéger de sa propre démesure, mais ces limites, il est seul à pouvoir se les donner. Il n'existe pas à l'extérieur de la communauté politique, dans une Église ou la conscience de chacun, des limites intangibles sur lesquelles on pourrait s'appuyer pour se protéger. Comme le dit fort bien Montesquieu, en cela plus républicain qu'on ne l'imagine, seul le pouvoir arrête le pouvoir. Un pouvoir qui ne connaît d'autres limites que celles qu'il se donne, c'est la définition donnée par Hobbes du pouvoir absolu. Hobbes s'écarte de la tradition républicaine en défendant deux thèses décisives : il loge ce pouvoir absolu dans une instance unique; il considère que cette instance unique peut être indifféremment un monarque ou une assemblée. La première thèse exclut le régime mixte. En ce sens, la première partie de cet exposé ne doit pas prêter à confusion : nous ne pouvons pas comprendre la relation de Hobbes à la tradition républicaine en faisant abstraction de sa critique du régime mixte. Le souverain doit être un. Cela exclut le partage de la souveraineté entre des instances distinctes. La seconde thèse vide la démocratie de son contenu républicain. Il n'importe plus que les citoyens aient part à la chose publique. Pour que la démocratie soit viable, il importe au contraire que la délibération conflictuelle sur les affaires communes soit refoulée hors de l'espace public.

Ces deux thèses nous indiquent à quelles conditions les républiques antiques sont des modèles. Il faut oublier ce qui, en elles, crée l'apparence du régime mixte, il faut oublier la délibération publique, il faut considérer que le peuple est l'unique monarque ${ }^{46}$. Puisque le pouvoir absolu réside en un seul, homme ou assemblée, le pouvoir absolu de type hobbésien ne peut être politiquement modéré que s'il se modère ${ }^{47}$. Hobbes compte sur l'intérêt bien compris des porteurs de la souveraineté. Le souverain qui use de son autorité illimitée pour exercer une puissance immodérée se heurte à la

45. Politique, liv. III, chap. III, 1276 b 1-b 12.

46. C'est la définition que donne Aristote de la démocratie la plus déviée, la plus proche de la tyrannie, voir Politique, liv. IV, chap. Iv, 1292 a 11.

47. De cive, chap. vII, $\$ 4$, note de l'édition de 1647 . 
résistance de ceux qui sont menacés dans leur vie et leur liberté de mouvement, dans la part inaliénable de leur droit naturel. Hobbes refuse que le pouvoir politique soit limité et contenu par un quelconque magistère moral ou religieux, il compte encore sur la nature qui en l'homme résiste à ce qui le détruit. Il reste nécessaire de faire appel à la nature parce que la souveraineté, telle que Hobbes la conçoit, manque d'un principe de limitation qui soit strictement politique. L'intérêt de la tradition républicaine, et avec elle de Montesquieu, est de permettre la recherche d'un tel principe.

Jean TERREL (janvier 1997). 